\title{
Multiplicidad e Integridad: \\ ¿Existe todavía una tendencia anti-evolutiva \\ en el Psicoanálisis Relacional? ${ }^{1}$
}

\author{
Donna Orange ${ }^{2}$ \\ Claremont, CA, USA
}

\begin{abstract}
A partir de Loewald, Mitchell y Bromberg, la autora defiende que las teorías deben sostenerse ligeramente ya que siempre necesitan correcciones. Revisa la teoría de los múltiples estados del self, y concluye que el psicoanálisis es un proceso de desarrollo, que crea un sentido continuo del self que actúa, no solo contenido en permanecer en los espacios.
\end{abstract}

Palabras clave: Múltiples estados del Self, Psicoanálisis, Giro ético.

From Loewald, Mitchell and Bromberg, the author argues that theories should be held slightly as they always need corrections. It reviews the theory of multiple states of the self, and concludes that psychoanalysis is a process of development, which creates a continuous sense of the self that acts, not only contained in standing in the spaces.

Key Words: Multiple States of the Self, Psychoanalysis, Ethical Turn.

English Title: Multiplicity and Integrity: Does an anti-developmental tilt still exist in relational psychoanalysis?

\section{Cita bibliográfica / Reference citation:}

Orange, D. (2017). Multiplicidad e integridad: ¿Existe todavía una tendencia anti-evolutiva en el Psicoanálisis Relacional?. Clínica e Investigación Relacional, 11 (2): 258-279. [ISSN 1988-2939]

[Recuperado de www.ceir.info ] DOI: 10.21110/19882939.2017.110204

\footnotetext{
${ }^{1}$ Versión revisada del trabajo leído por la autora en la Conferencia Internacional de IARPP celebrada en Roma, 2016. Traducción castellana de N. Monserrat Gómez García.

2 Donna Orange - 570 Mayflower Road, Claremont, CA 91711, USA. Contacto: Donna.orange@gmail.com Más información en: www.donnaorange.net
} 
A William James le gustaba recordarnos que nada es tan práctico como una buena teoría. Pero nuestras teorías, tal como su amigo Charles Sanders Peirce enseñó implacablemente ${ }^{1}$, necesitan sostenerse ligeramente ya que siempre necesitan correcciones. La "contrición a la falibilidad" en el que los pragmáticos creían nos sirven mucho cuando estudiamos la historia del psicoanálisis, incluyendo la corta historia de los enfoques relacionales. Viene con un optimismo robusto el que siempre podemos aprender más, tanto de nuestros pacientes como de los demás, dentro de una comunidad de académicos que aceptan a aquellos que hablan varios idiomas y que enseñan de maneras que al principio no apreciamos, verbal y no verbalmente. En nuestros intentos por mejorar nuestras teorías, podríamos cometer pasos en falso, pero siempre podemos volverlo a intentar.

\section{Introducción}

Aquí describiré los aspectos relacionados con la teorización de Stephen Mitchell y Philip Bromberg, sugiriendo donde considero que yacen los problemas residuales y también una solución tentativa usando los recursos de dos de mis disciplinas, la filosofía y el psicoanálisis.

Cuando fallece Stephen Mitchell, justo había completado una década de estudio intensivo sobre la obra de Hans Loewald, su escritor psicoanalítico favorito. Su relectura de Freud es evidente no sólo en los primeros dos capítulos de Relacionalidad (S.A. Mitchell, 2000), sino en todo su desarrollo. Mitchell, autor de la expresión despectiva: "inclinación del desarrollo", se había contradicho a sí mismo, pareciendo ya no estar preocupado por infantilizar a los pacientes y ahora -con Loewald, Bowlby y Winnicott- vislumbró al psicoanálisis como un proceso de desarrollo. ¿Qué ha sido de la contradicción de Mitchell desde su muerte? Quizá el pensamiento clínico de Jody Davies, con su receptividad a las voces jóvenes del paciente, así como al trabajo winnicotiano de Slochower y Ogden, han recogido esta contradicción. Sin embargo, el tan popular giro de hablar sobre los "múltiples estados del self" lo veo como algo ambiguo y problemático. Mientras avanza nuestro sentido de la complejidad de la experiencia y reemplace la simple conversación de regresión, también puede obstruir una sensibilidad del desarrollo como la que tienen en común Loewald, Winnicott y Kohut, todas las voces necesarias en el coro relacional.

Sugeriré más adelante que esta sensibilidad compartida del desarrollo, hacia la cual Mitchell estaba firmemente volviendo en sus últimos años y que se ha desvanecido nuevamente desde que lo perdimos, es un elemento fundacional del "giro ético" en psicoanálisis, siendo escrito por Cushman (Cushman, 2007), Layton (Layton, 2009), Leary 
(Leary, 2000), (Baraitser, 2008), entre otros. El psicoanálisis como proceso de duelo e integración se relaciona muy cerca con la integridad moral y el coraje.

En este contexto he mencionado el desdén por un cuidado ético en algunos círculos relacionales, así como las excluyentes actitudes de "no suficientemente relacional" y "eso no es psicoanálisis" que traicionan la inclinación anti-desarrollo. En contrate, mi ensayo abogará por compasión hacia el niño vulnerable y que sufre dentro de nuestros pacientes y en nosotros mismos, así como hacia el mundo en llamas dentro del cual vivimos y morimos.

\section{Mitchell: la influencia de Loewald y el giro del desarrollo}

Como muchos analistas entrenados bajo la tradición interpersonal, el joven Stephen Mitchell nunca perdió el amor en los desarrollistas como Winnicott y Kohut. Aunque la crítica comenzó en su revolucionario libro con Jay Greenberg (Greenberg \& Mitchell, 1983), Mitchell publicó su ahora famoso trabajo (S.A. Mitchell, 1984) sobre "la inclinación del desarrollo", haciendo sus objeciones más claras, justo unos meses después. Por "inclinación del desarrollo" él se refería a "estrategia acomodaticia," (en el libro "modelo-mixto"), esto es, mantener la teoría pulsional y el complejo de Edipo intactos, pero instalando las relaciones objetales más tempranas y profundas ${ }^{2}$. Para él, los primeros practicantes de este acercamiento ahora incluían a Klein, Balint, Mahler, Guntrip, Winnicott y Kohut. Estos teóricos vieron las necesidades relacionales como infantiles y regresivas. Su práctica, desde la perspectiva del joven Mitchell, tendían a ver a los pacientes como víctimas pasivas de una temprana privación y a los analistas como buenos proveedores. Desde el punto de vista de Mitchell, los teóricos más consistentes que él prefería -Fairbairn, Sullivan y Bowlby -comprendieron las necesidades relacionales-y necesidades aún más maduras de la dependencia-como simplemente humanas. Desafortunadamente, él pensaba, su teorización perdía toda la elegancia y complejidad de la teoría Freudiana y la tradición. En 1984, antes del total desarrollo de su propia perspectiva relacional, Mitchell simplemente expuso este inconveniente con gran claridad; él aún no sugería su concepto alternativo.

En los próximos años, los libros más importantes de Mitchell (S.A. Mitchell, 1988, 1993, 1997) se desarrollaron en la matriz relacional como la base clínica y teórica de lo que se volvería a llamar como psicoanálisis relacional. En 1987 lo definió así:

El modelo relacional cae en la premisa de que los patrones repetitivos dentro de la experiencia humana no son debidos, como en el modelo pulsional, a una búsqueda de placeres secretos (ni tampoco, como en la comprensión de Freud en su publicación de 1920, de los trabajos automáticos del instinto de muerte), sino de una profunda 
tendencia a preservar la continuidad, conexiones y familiaridad del propio mundo personal. Hay una poderosa necesidad por preservar un sentido duradero de uno mismo como asociado con, colocado en términos de, relacionado a, una matriz de otras personas, ambas en términos de transacciones actuales, así como de presencias internas. Las configuraciones relacionales básicas tienen, por definición, tres dimensiones-el self, el otro y el espacio entre ellos. No hay "objeto" desde un significativo sentido psicológico, sin cierto sentido particular de uno mismo en relación a ello. No hay "self" dentro de un significativo sentido psicológico, en aislamiento, fuera de la matriz de relaciones con los otros. Ni el self ni el objeto son conceptos significativamente dinámicos sin presuponer cierto sentido del espacio psíquico en el cual interactúan, en donde ellos hacen cosas con o entre ellos. Estas dimensiones están entrelazadas de manera sutil, tejiendo conjuntamente la experiencia subjetiva y el mundo psicológico del analizante. (S.A. Mitchell, 1987, p. 403).

La palabra matriz aparece dos veces aquí: "una matriz de otras personas" y "la matriz de relaciones con los otros", como si la matriz fuera siempre contemporánea. Pero más tempranamente, en discusiones de Kohut, Mitchell había usado la expresión "matriz maternal-infante" (S.A. Mitchell, 1979) y de Winnicott, "matriz infante/madre" (S.A. Mitchell, 1983). Por lo que su capacidad para absorber el profundo psicoanálisis del desarrollo de Hans Loewald y para identificar su matriz relacional con la "densidad primordial" de Loewald, no debería sorprendernos.

Es argumentable lo que se ha desarrollado desde entonces en el psicoanálisis contemporáneo-ya sea que utilice este lenguaje o no-manteniéndose en relación con esta idea de la matriz relacional3. Es fácil referir el trabajo de aquellos que escribieron de la terceridad de una manera u otra (Aron, 2006; Benjamín, 2004; Gerson, 2009; Knoblauch, 1999; Muller, 1999; T.H. Ogden, 1994), ya que claramente estos autores difieren enormemente en su uso de este término. (Ninguno de estos autores, sin embargo, a mis oídos, le da a la terceridad el profundo desarrollo que la matriz de Mitchell tenía al final de su vida). Además, las penetrantes suposiciones del compromiso mutuo, participación y responsabilidad en el análisis deben su origen a la matriz relacional de Mitchell. Su invisible influencia podría impregnarnos tan insistentemente como la de Freud.

Su voz pionera, sin embargo, podría enmascarar su silencioso camino como un devoto y erudito estudiante de la historia del psicoanálisis, uno que emergió claramente en su Relacionalidad, un libro que pareció muy cercano a su muerte que la mayoría de nosotros nunca tuvimos la oportunidad de conversar con él acerca de los cambios en su pensamiento que señalaba. Él dedicó los primeros dos capítulos de este libro al trabajo de Hans Loewald, 
cuyo trabajo había leído y releído por más de una década (S. Mitchell, 2004) con gran "placer." Nos dijo que Loewald se había convertido en su "escritor psicoanalítico favorito." ¿Quién era este Loewald y quien era él para Mitchell?

Loewald recogió los brillantes fragmentos del pensamiento de Freud-trabajos que Freud había dejado por ahí mientras ingeniosamente confrontaba nuevos acertijos clínicos y teóricos-y usaba lo que aprendía de Heidegger para integrarlos dentro de un todo ${ }^{4}$. Loewald nos veía como siempre intentando re-encontrar nuestra original experiencia unitaria, nuestra "densidad primordial"-la misma que Winnicott estudió como "no hay tal cosa como un infante" - del cual nosotros después diferenciaremos a medida que nos volvemos nosotros mismos en relación con los otros. (Balint lo hubiera llamado la "mezcla harmónica interpenetrante" (Balint, 1992)). Loewald reinterpretó la teoría pulsional tal y como la biología evolutiva contemporánea lo hace, no como una teoría de conflicto, sino como Eros (Lear, 1998), un esfuerzo por recuperar la unidad original. Los instintos para él emergen desde la integración del infante y el ambiente. Conceptos básicos en la teoría psicoanalítica pierden su extrañeza a manos de Loewald-en parte porque leyó y pensó en el alemán de Freud y constantemente intentaba escapar de lo que podríamos llamar la edición estandarizada. La "catexia" se vuelve una actividad organizadora, vinculante y algunas veces hasta amor. El "proceso primario" se vuelve la riqueza de nuestro continuo acceso a nuestras más tempranas e incorporadas experiencias entre madre y niño, que constantemente se alterna con la diferenciada y más organizada vida lingüística. Lo "secundario" desde la perspectiva de Loewald gana la connotación de secundario como llegando después en el proceso de la organización psicológica y de la integración de la experiencia. El "proceso primario" pierde completamente vergüenza y se convierte en el recurso rico en la vida imaginativa, cultural, e inclusive, tal vez, trascendente. Un cercano lector de Freud que nunca abandonó el psicoanálisis americano ni fundó una escuela de pensamiento, Loewald reconoció a los observadores de bebés y a Winnicott como sus espíritus afines.

No es de extrañar que, como fenomenólogo, Loewald haya replanteado profundamente la temporalidad. Pasado, presente y futuro se compenetran tanto que él podría dar la bienvenida al proceso primario como la fuente de nuestra creatividad y vida religiosa. (En repetidas ocasiones, expresó su pesar de que Freud pudiera ver poco uso constructivo de la religión).

¿Cómo logro Loewald todo este replanteamiento? Nachträglich, esto es, al revés. Él cogió un posterior texto de Freud, de Civilización y sus Descontentos, y lo utilizó para leer todo lo que vino antes, como para decir, "Aquí está el significado profundo en Freud que él hubiera desarrollado si hubiera tenido más tiempo." Freud escribió: 
Un infante en el pecho aún no distingue todavía su Yo del mundo externo como la fuente de las sensaciones que fluyen sobre él/ella. Él/Ella gradualmente aprende a hacerlo, en respuesta a varias incitaciones... De esta manera, entonces, el ego se separa a si mismo del mundo externo. O, para ser más precisos, el ego incluye originalmente todo, después se separa de un mundo externo de símismo. (Sigmund Freud \& Strachey, 2005, pp. 66-68).

Con este texto en mano, Loewald relacionalizó a Freud y argumentó en su famoso trabajo $(\mathrm{H}$. W. Loewald, 1960) que el poder terapéutico del psicoanálisis es resultado de lo relacional, es decir, transferencial de viejas miserias. El analista, lejos de un espejo desapegado y distante, se vuelve a si mismo disponible para el paciente para el proceso de transferencia del desarrollo del Yo, del cual Loewald escribe:

Sin esa transferencia-de la intensidad del inconsciente, de los modos infantiles de experimentar la vida sin lenguaje y con poca organización, pero con la indestructibilidad y el poder de los orígenes de la vida-hacia el preconsciente y a la vida diaria presente y a sus objetos contemporáneos-sin tal transferencia, o hasta el punto en el cual tal transferencia se frustrase, la vida humana se vuelve estéril y una cáscara vacía. Por otro lado, el inconsciente necesita de la realidad externa actual (los objetos) y la realidad psíquica actual (el preconsciente) para su propia continuidad, para que no sea condenada a vivir la vida sombría de los fantasmas o de destruir la vida (Loewald, 2000, p. 250).

El pasaje más famoso de este artículo, tan importante para Mitchell que nombró después de su artículo sobre Loewald (S. A. Mitchell, 1998), siempre merece la pena releerlo:

La neurosis de transferencia, en el sentido técnico del establecimiento y resolución de ella en el proceso analítico, es debido a la sangre de reconocimiento que se le es dado inconscientemente al paciente para probar -de modo que los viejos fantasmas puedan resurgir a la vida. Aquellos que conocen fantasmas nos cuentan que ellos añoran ser liberados de su vida fantasmal y llevados a descansar como ancestros. Como ancestros viven adelante en la generación actual, mientras que como fantasmas están forzados a atormentar a la generación actual con su vida sombría. La transferencia es patológica hasta donde el inconsciente es una multitud de fantasmas [mi definición favorita de inconsciencia], y este es el comienzo de la neurosis de transferencia en el análisis: los fantasmas del inconsciente, atrapados por defensas, pero atormentando al paciente en la oscuridad de sus defensas y síntomas, están permitidos a probar la sangre, están dejados sueltos. En la luz del análisis los fantasmas del inconsciente son colocados y puestos a descansar como ancestros cuyo poder es tomado y transformado en la 
intensidad más reciente de la vida presente, del proceso secundario y objetos contemporáneos (Hans W. Loewald, Loewald, \& Loewald, 2000, pp. 248-249).

Primero, como los investigadores de niños, en particular Daniel Stern (Stern, 1985) Loewald creía que nosotros nos organizamos y sanamos a nosotros mismos a través de la "internalización" especialmente en las áreas que los freudianos hablan en la región del desarrollo del superyó. Ya no se ve obligado como un autómata, el niño o adulto en crecimiento ha hecho valer sus propios valores morales: "Un sentido del Yo comienza a emerger con una internalización cada vez mayor, llevando al desarrollo de un sentido de autoresponsabilidad con la formación del superyó y el hombro de la culpa" (H. W. Loewald, 1985, p. 437). En análisis, creía él, el trabajo de duelo esta indispensablemente ligado a la reanudación de los procesos de internalización. "La renuncia a los objetos externos y su internalización implica un proceso de separación, de pérdida y de restitución que de muchas maneras es similar al duelo. Durante el análisis, los problemas de separación y duelo se manifiestan de manera específica en momentos de interrupción y, más particularmente, en las fases terminales del tratamiento." (H. Loewald, 2007, p.1114). El duelo internaliza lo que debe ser abandonado e integra una vida personal, una vida moral, como él diría más tarde.

Ahora, en la década de 1990, además de Sullivan y Fairbairn, Michell había encontrado un tercer (después de Sullivan y Fairbairn) teórico proto-relacional, uno que se había convertido en su "escritor psicoanalítico favorito". Loewald había vinculado todas las experiencias dispares con las cuales analistas y pacientes batallaban con la unidad original, y entendían el proceso analítico en sí como vinculante. Mitchell condenso elegantemente la descripción de Loewald sobre la psicopatología como la densidad principal perdida:

...desde el punto de vista de Loewald, la psicopatología, más ampliamente concebida, representa un desequilibrio entre fuerzas centrífugas y centrípeta de la mente. En la psicosis, la densidad primal socava la capacidad de hacer distinciones adaptativas y normativas entre el interior y el exterior, el self y el otro, la realidad y la fantasía, el pasado y el presente. En la neurosis o, como sugiere ocasionalmente Loewald, la adaptación normativa a nuestro mundo cientificista e hipertecnológico, los constituyentes de la mente se han alejado demasiado de su unidad original densa: lo interior y lo exterior se convierten en dominios separados e impermeables; El self y el otro se experimentan aislados unos de otros; La realidad esta desconectada de la fantasía; Y el pasado se ha alejado de un presente superficial y sin pasión. (S. A. Mitchell, 1998, p. 826). 
Mitchell, que restauró la continuidad con Freud por su inmersión en Loewald, comenzó a estudiar seriamente la teoría del apego y a leer a Winnicott con un oído para "el núcleo del individuo en una aislada privacidad" (S. A. Mitchell, 2000, p. 87), para un sentido similar del desarrollo de un individuo, así como para las diferencias concernientes con la ilusión y la fantasía.

\section{Bromberg sobre los múltiples estados del self.}

Incluso antes de que Greenberg y Mitchell sacudieran el mundo para muchos de nosotros o, mejor dicho, nos dieran una manera de pensar sobre nuestras dudas y esperanzas, Bromberg estaba escribiendo sobre la regresión (P. M. Bromberg, 1979)- una actividad subversiva para un interpersonalista y una pregunta del desarrollo ya sea considerada clásicamente o desde un punto de vista de las relaciones de objeto-y que corresponde, como ahora sabemos, con el radical Merton Gill (P. Bromberg, 2011). También escribe ( $P$. Bromberg, 2013) de su afecto por Mitchell, y podemos suponer, de Mitchell para él. En esta coyuntura temprana, Bromberg parece haber visto la regresión como una indicación casi precisa de la visión del desarrollo del psicoanálisis que le atribuyo a Loewald y posteriormente a Mitchell:

Pero, independientemente de cuán "profunda" sea la regresión, sugiero que la regresión en el sentido que uso aquí no es un concepto limitado a pacientes analíticos que tienen un deterioro grave del $\mathrm{Yo}$, sino que es un componente fundamental del psicoanálisis en general y del enfoque interpersonal en particular. El Yo (o self) para crecer, debe voluntariamente permitirse a sí mismo el volverse menos intacto-a la regresión. Empíricamente, esta es una forma de definir la regresión en el servicio del Yo (P. M. Bromberg, 1979, p. 653). 5

Formados en el Instituto William Alanson White, Mitchell y Bromberg a finales de los años setenta y principios de los ochenta estaban trabajando su camino hacia la integración de las perspectivas psicoanalíticas con inicios similares. Adrienne Harris escribe sobre este periodo:

Uno de los rompecabezas ${ }^{6}$ más fascinantes para mí ha sido el cambio radical en Mitchell en abordar nuevamente los problemas del desarrollo y de la experiencia temprana, esto es, su alejamiento, al final de su vida, desde la inclinación antidesrrollo de su primer trabajo relacional. Veo ahora que el trabajo de Bromberg sobre la regresión fue parte del preámbulo de esos cambios en Mitchell. Ciertamente más adelante, la reaparición de Bowlby y de la teoría del apego jugaron un papel en los cambios en las preocupaciones de Mitchell, pero lo que Bromberg estaba 
haciendo, podemos ver, era crucial para su propia obra, pero también creo que contribuyó a los cambios en Mitchell también (Harris \& Bass, 2011, p.242).

Al igual que la mayoría de los lectores de la última obra de Mitchell, Harris omite o ignora a su "escritor psicoanalítico favorito" (Loewald)7. Aun así, ella hace una sugerencia fascinante: la posibilidad de que Bromberg plantara algo en sus conversaciones con Mitchell, algo que Mitchell desde el principio no pudo soportar, pero luego lo hizo con venganza, y algo que el mismo Bromberg posteriormente minimizó a favor del concepto menos desarrollado de la disociación. Ya sea que ella pudiera ver en su propia idea lo que hago, no puedo decirlo, sin embargo, me parece fructífero para rastrear lo que parece haber ocurrido.

Más allá de su popularidad, la teoría de los estados múltiples del self (P. M. Bromberg, 1992, 1994, 1996) se ha convertido en algo axiomático en el psicoanálisis relacional contemporáneo, en disciplinas como los estudios religiosos (Cooper-White, 2007). En una metáfora asombrosa, Bromberg imagina regiones diferentes y disociadas en el paciente a las que el analista gana pasaportes. La experiencia básica a lo que se refiere esta teoría, descrita originalmente por Harry Stack Sullivan, rechaza emociones, motivaciones, acciones o residuos traumáticos particulares como "¡no-yo!".

Bromberg explica los objetivos para este tratamiento:

El funcionamiento mental óptimo consiste en que una persona sea capaz de acceder a múltiples estados del self en forma conflictiva y el tratamiento psicoanalítico debe proveer un contexto favorable para facilitar la comunicación interna entre estados disyuntivos que mantienen aislados unos de otros disociativamente (P. Bromberg, 2009, p. 350).

Los escritores de psicoanálisis clínico y aplicado han usado este concepto con frecuencia en los últimos años, por ejemplo (G. Bass, 2002; Chefetz, 2003; J. Davies, 196; Harris, 1996; Hirsch, 1997; Yerushalmi, 2001), sin prestar mucha atención a lo que puede perderse, o a su supuesto corolario de que dicha conversación implica que no existe una personalidad central, organizada o responsable. Sin embargo, Bromberg dijo, al menos en los primeros años de su teoría, de otra manera:

Contamos con la existencia en el paciente de una personalidad nuclear cohesiva que nos parece a nosotros y al paciente más o menos parecida a la misma persona sin importar los cambios de un momento a otro de los estados del self, de las alteraciones en el funcionamiento mental o, inclusive, de la inesperada emergencia del fenómeno disociativo que Sullivan (1953) nombró como experiencia "no-yo" (P.M. Bromberg, 1991, p. 403). 
Las cosas se vuelven confusas, tanto fenomenológicamente como clínicamente, cuando se producen divisiones extremas, con amnesia, como lo demuestran quienes trabajan clínicamente con supervivientes de tortura y combate, abuso infantil extremo, genocidios y otras situaciones similares. ¿Seguimos hablando de múltiples estados del self tal y como estos autores atribuyen tan fácilmente a todos nosotros? ¿Dónde está la personalidad unificadora cuando el cónyuge y los hijos dicen "ya no lo conozco"? ¿Qué pasa con la paciente que regresa al día siguiente y no recuerda nada de la sesión del día anterior cuando había revivido una violación grupal? ¿Quién es el paciente y quién es el responsable de lo que este paciente promete, hace y dice? Todas estas preguntas surgen cuando imaginamos que existe, o no, una personalidad central. Además, por supuesto, Bromberg y todos aquellos a los que ha inspirado han desarrollado reflexiones extremadamente útiles sobre los procesos intersubjetivos involucrados en muchas formas de disociación, especialmente las que vemos en el trabajo clínico.

Pero debemos señalar que estas preguntas se encuentran en diferentes ámbitos del discurso: (1) las disputas filosóficas y éticas sobre la identidad humana que John Riker (Riker, 2013) revisa para nosotros y que Charles Taylor expuso tan magistralmente en su Fuentes del Self ${ }^{8}$, (Taylor, 1989); (2) las preguntas especulativas (metapsicológicas) acerca de lo que está detrás de los fenómenos clínicos—una totalidad o un motor primario unitario, un cúmulo de estados del self, ambos, una organización emergente de la personalidad, y así sucesivamente, y (3) los mismos fenómenos clínicos experienciales, como describibles por ambas partes. Creo que nuestras dificultades están en nuestro fracaso por distinguirlas.

Las distinciones, sin embargo, son la mercancía de intercambio para los filósofos, así que aquí vamos. Veamos primero la disociación, una palabra utilizada al menos desde Pierre Janet. En el psicoanálisis actual ha llegado a tener un cúmulo de significados que Ludwig Wittgenstein habría descrito como "parecido familiar"9. Desde la amnesia completa después de un accidente automovilístico, hasta la pérdida de memoria mientras se viaja porque se preocupa por otro problema, hasta el rechazo de aspectos de la vida emocional de uno como "no-yo"-todos estos fenómenos y más se denominan disociación. La teoría de los múltiples estados del self, diría yo, cae en una trampa lógica cuando argumenta desde un cúmulo de fenómenos disociativos que tienen solo un "parecido familiar" entre sí, como si pudiéramos atribuirles este concepto a todos, y luego las razones por las cuales los humanos no pueden, y no deberían, esforzarse por una integración suficientemente buena de la personalidad. Este modo de pensamiento clínico tiende a sugerir que el diálogo con nosotros mismos no puede conducir a algún sentido de que "esto es lo que creo", y "estas cosas son importantes para mí." Volveremos a los problemas éticos generados por este "no puedo". 
En segundo lugar, vamos a volver a la evidencia adjudicada a la teoría de los múltiples estados del self.

Bromberg escribe:

Existe ahora abundante evidencia de que la psique no comienza como un todo integrado, sino que es de origen no unitario-una estructura mental que comienza y continúa como una multiplicidad de estados del self que en su maduración alcanzan un sentimiento de coherencia que sobrepasa la conciencia de la discontinuidad (Bromberg, 1993, p. 162). Esto conduce a la experiencia de un sentido cohesivo de la identidad personal y la ilusión necesaria de ser "uno mismo." (P. M. Bromberg, 1994, p. 521).

¿Y que es, exactamente, esta "abundante evidencia"? Continúa:

Para los psicoanalistas, esta concepción de la mente ha sido apoyada por los estudios infantiles orientados psicoanalíticamente como los de Emde, Gaensbaure y Harmon (1976), Sander (1977), Stern (1985), Woldd (1987) y Beebe y Lachmann (1992), pero el apoyo más directo ha venido de las investigaciones empíricas no-analíticas sobre el funcionamiento mental normal y patológico del adulto-investigación que representa una amplia gama de disciplinas y centros de investigación (p. 521)

Después continúa citando los estudios de Frank Putnam (Putnam, 1988) sobre disociación extrema que ahora serían diagnosticados como Desorden Disociativo de la Identidad y Desordenes Complejos de Estrés Post-Traumático, y argumenta de estos que la mente es esencialmente múltiple y la auto-experiencia ilusoria.

El argumento de la multiplicidad contemporánea es persuasivo porque establece la continuidad entre la psicopatología extrema y las experiencias humanas cotidianas, como lo hizo Freud cuando inventó el psicoanálisis. El sufrimiento dramático y traumático se acercan a nuestras luchas ordinarias. "Somos simplemente más humanos que otra cosa." (Sullivan \& Mullahy, 1948, p. 16). Pero en ese caso quizás necesitamos oír la voz de Wittgenstein recordándonos que el rey en el ajedrez y el rey de Inglaterra funcionan muy diferente, aunque compartan el mismo nombre. No sólo la disociación podría ser una cuestión de semejanzas familiares, sino también experiencias de la multiplicidad en general.

Además, la "abundante evidencia" de los estudios de desarrollo para la mente como múltiples estados concurrentes del self, y la salud mental definida como la capacidad para "estar en los espacios" entre ellos, necesitan mirarse con atención. Desafortunadamente, Daniel Stern y Lou Sander ya no se encuentran entre nosotros, pero podemos consultar sus trabajos. El trabajo clásico de $\operatorname{Stern}^{10}$ (Stern, 1985) concierne enteramente al desarrollo humano en un sentido del self, y para mi lectura, no contiene ninguna indicación de que en 
ese momento habría aceptado que los humanos deberían renunciar al proyecto de integración personal. Si, él difería con Winnicott y Loewald con la unidad original (oneness), pero como ellos, visualizaba al ser humano siempre en busca de integración. Su trabajo posterior, más fenomenológico, sobre el impacto evolutivo del encuentro de momento presente y sobre los efectos de la vitalidad, así como su liderazgo en el Grupo de Estudio de los Procesos de Cambio de Boston, complejizaba, pero no abandonaba su trabajo anterior.

Lou Sander, un grande en la investigación infantil, trabajó, al igual que Bromberg, desde la perspectiva de los sistemas no lineales de auto-organización y las teorías de la complejidad. Para Sander, estas teorías explicaban mejor lo que él veía en el desarrollo de la identidad emergiendo del reconocimiento (Sander, 1995), y también concordando con lo que había aprendido de Winnicott:

No conozco una mejor descripción de un proceso de reconocimiento que aquel proceso que Winnicott (1972) describe. Él describe, e ilustra con muchos ejemplos de caso, el proceso interactivo entre el terapeuta y el niño que continúa mientras cada uno alterna dibujos en el juego que él llama "garabato." Winnicott detalla los dibujos mediante los cuales cada uno embellece el garabato del otro, dentro del contexto de las observaciones de Winnicott, para llevarlos a un momento de conciencia compartida a medida que el niño se da cuenta de que el otro es consciente de lo que el niño es consciente en su interior. Este es un momento de especificidad en el reconocimiento, el cual Winnicott llamo el "momento sagrado" - un "momento de encuentro" que implica una nueva coherencia en el niño sobre la experiencia de su mundo interno y externo de consciencia (awareness). Las consultas que Winnicott describe son a menudo sesiones únicas de diagnóstico, pero si se alcanza el "momento sagrado" de ser "conocido", se produce un cambio en la organización autorreguladora del niño que ha sufrido durante muchos años, incluso desde esa experiencia única. La recurrencia de tales momentos provee las condiciones dentro de las cuales uno llega a "conocerse" como uno es "conocido". Con la recurrencia y la construcción inherente de expectativa del cerebro, es un pequeño paso hacia la definición de identidad de Erikson (1950) como una "confianza en que la igualdad y la continuidad interiores son iguales por la similitud y continuidad del significado de uno para los demás" (p. 228).

Gradualmente, como Winnicott y Sander creían, en condiciones del desarrollo suficientemente buenas, un sentido de un "verdadero self" - en contraste con el "falso self" de complacencia-prevalecería.

Donde Bromberg generaliza de sus observaciones "la no linealidad normal de la mente humana" (P. M. Bromberg, 1996, p. 529), y cree que clínicamente "lo que se requiere es que 
las múltiples realidades que están siendo sostenidas por diferentes estados del self encuentren oportunidades para la vinculación" (p. 543), Sander encuentra una investigación relacional para toda una vida de la identidad Eriksoniana. Parece una extensión para citar su pensamiento como evidencia de las opiniones de Bromberg.

El pensamiento de Beebe y Lachman, cercanamente relacionados con las ideas de Stern y Sander, describen similarmente el desarrollo de la coherencia e incoherencia de la experiencia del self dependiendo en la situación relacional del niño. En contraste con la teoría de los múltiples estados del self, Lachmann arroja una teoría clínica que supone:

Un modelo procedimental del self como singular, esforzándose por integrarse y temporalmente continuo. Este self nunca es estático, sino que se actualiza constantemente en las interacciones que requieren la regulación de los afectos, la excitación y las percepciones en un contexto y con un trasfondo de regulación mutua, sensible y contingente. Desplazándose en diferentes contextos, el self integrado puede priorizar experiencias, abrazar una serie de conflictos y tolerar estados afectivos dispares (Lachmann, 1996, p. 610).

Claramente Lachmann, al igual que Stern y Sander, encuentra en la investigación infantil "abundante evidencia" para la compleja experiencia del self que siempre está en proceso de integración, pero rechazaría los usos que Bromberg y otros partidarios de la teoría de los múltiples estados del self están poniendo en su trabajo. (En realidad, creo que Bromberg encuentra afinidad con estos grupos de investigadores infantiles no porque proporcionen evidencia para la teoría de los múltiples estados del self, sino porque él mismo nunca abandonó realmente una perspectiva del desarrollo, ej. (Philip M. Bromberg, 1998, p. 90).

\section{Problemas con la teoría de los múltiples estados del self.}

Más allá de las debilidades en los argumentos aducidos para apoyar esta teoría, creo que tiene serios problemas inherentes: 1) Regresa al psicoanálisis relacional, aunque involuntariamente, a su temprana inclinación anti-desarrollo; 2 ) evade los problemas de la finitud humana y el luto; y lo peor de todo, 3) falla en fundamentar y apoyar la subjetividad ética. Estos son cargos muy serios, así que vamos a llenarlos.

1) Con importantes excepciones (A. Bass, 2009; J. Davies, 2009; Grand, 2010; D. M. Orange, 2011; Slochower, 2013) $)^{11}$, me parece que el psicoanálisis relacional ha vuelto en gran medida a una perspectiva anti-desarrollo de finales de los ochentas y han olvidado el movimiento radical de los últimos años de Mitchell. La adopción de una perspectiva del desarrollo con Winnicott, Loewald, Kohut, Bowlby, Fairbairn y el 
Mitchell en sus últimos años, significa ver el proceso analítico en sí mismo como un proceso de desarrollo. En palabras de Heinrich Racker:

Termino esta sección sobre la posición interna del analista diciendo que sólo se puede esperar que el paciente acepte la re-experiencia de la infancia si el analista está dispuesto a aceptar plenamente su nueva paternidad, a admitir el afecto por sus nuevos hijos/as y luchar por una nueva y mejor infancia 'invocando todas las fuerzas mentales disponibles' (S. Freud, 1917). Su tarea consiste idealmente en un interés constante y vivo y una continua empatía con los acontecimientos psicológicos del paciente, en un análisis metapsicológico de cada expresión y movimiento mental, en su principal atención y energía siendo dirigida hacia la comprensión de la transferencia (hacia la siempre presente 'nueva infancia') y superar sus aspectos patológicos mediante interpretaciones adecuadas (Racker, 1968, p. 33).

Parece seguro decir que el psicoanálisis contemporáneo (los relacionales inspirados en Mitchell, los psicólogos relacionales del self, freudianos contemporáneos, entre otros) generalmente están de acuerdo con Racker, aunque las actitudes de los desarrollistas pueden expresarse de manera diversa. Los analistas empiezan a escribir sobre el "amor analítico" (D. Shaw, 2003) y a recordar a Sandor Ferenczi no solo por sus experimentos de análisis mutuo, sino por su defensa plena hacia el niño en el paciente adulto (S. Ferenczi, 1931, 1949).

La teoría de los múltiples estados del self también reconoce que muchos pacientes se disocian ${ }^{12}$, es decir, se dividen para hacer frente a un trauma relacional, a menudo de origen del desarrollo, que con mucha frecuencia es transmitido transgeneracionalmente. De hecho, la teoría pretende explicar cómo los pacientes y los analistas evocan y catalizan las divisiones del otro. La adhesión oficial de la teoría hacia la temporalidad no lineal combinada con la doctrina del aquí y el ahora heredada del psicoanálisis interpersonal, sin embargo, hace dificil ver el proceso analítico en sí como del desarrollo. Si el desarrollo requiere un concepto lineal de tiempo-como no lo hace Stern, Sander, Beebe y Lachmann o Loewald-esta teoría no puede apuntar más que a permanecer en los espacios ( $P$. M. Bromberg, 1996) entre los estados del self de uno mismo.

Por otra parte, como dice Rich Cheftez (Cheftez \& Bromberg, 2004), y me pregunto, especialmente leyendo a Sue Grand (Grand, 2000), si tal "espacio intermedio" toma los estados traumáticos extremos del alma asesinada (Shengold, 1989) o ahogada (Levi, 1988) en serio. Ya sea que trabajemos con los agresores como Grand y otros colegas, o con las víctimas de narcisismo maligno (Daniel Shaw, 2014) que se creen benevolentes, pero nos tratan 
cruelmente, si surgen cuestiones de responsabilidad que estiran la teoría de los múltiples estados del self.

Pero ¿qué pasaría si el desarrollo, como Stern, Sander, Beebe Y Lachmann creían con el clásico de Thelen y Smith (Thelen \& Smith, 1996), es en sí mismo no lineal y complejo? ¿Qué pasaría si el Nachträglichkeit (acción diferida) ${ }^{13}$ que nos dejó Freud y tan ampliamente estudiado hoy (Birksted-Breen, 2003; Dahl, 2010) puede ahora entenderse como una Nachträglichkeit (acción diferida) intersubjetiva, sin la completa perdida de la búsqueda de integración personal e integridad ética que parece tan frágil en el mundo de ahora? Este tema merece más desarrollo que el espacio lo permite aquí.

2) El duelo y el reconocimiento de la finitud (Hoffman, 2000; Stolorow, 2007) requiere de cada uno de nosotros una mayor integración de simplemente dejar que los estados del self co-existan. El duelo significa darse cuenta de que las personas y los lugares y los aspectos de mí mismo han sido realmente e irreversiblemente perdidos, salvando y atesorando lo que puedo, y dejando marchar el resto. Freud comenzó a enseñarnos sobre esto; Loewald y Mitchell lo continuaron. El aceptar la finitud, la muerte y la vulnerabilidad, significa que alguien sabe que los muchos "no yo", incluyendo aquellos generados en años anteriores (S. Ferenczi, 1949), pertenecen a alguien que no va a durar para siempre, y mientras tanto tiene un futuro y una capacidad limitadas, incluso mientras se expande en otros aspectos.

3) ¿Cómo puede alguien que permanece entre los espacios—el resultado idealizado de mucho psicoanálisis relacional contemporáneo-tomar una postura ética ante la injusticia? Incluso los psicoanalistas contemporáneos cargan con el legado de aquellos que afirmaban que desconocían lo que el régimen Nazi o el régimen de Pinochet estaban haciendo. Del mismo modo que los psicoanalistas, como muchos de nuestros compañeros ciudadanos, vivimos en una cómoda burbuja de "múltiples estados del self" que llamo doble consciencia (D. Orange, 2016a), sabiendo y no sabiendo que estamos destruyendo tanto nuestra tierra e inclusive antes, a nuestros hermanos y hermanas más pobres. Una teoría que valora el mantener conciencias divididas y ridiculiza la búsqueda de integridad personal y un estado del self integrado sólo porque con frecuencia nos sentimos "con dos mentes", podría fallar a la crucial prueba de valor civil. Dudo que los colegas que hablan sobre los múltiples estados del self hayan pensado verdaderamente bien las implicaciones éticas y políticas de esta teoría.

Sin embargo, cada vez más escuchamos voces dentro del psicoanálisis relacional que Iloran por justicia social: para aquellos marginados por el género y la orientación sexual, por la 
pobreza y la raza, por la exclusión de cualquier tipo. Nos sentimos más y más llamados a ver y escuchar a aquellos otros para nuestra posición privilegiada que nos mantienen ciegos y sordos, y a nuestras propias implicaciones en sus demandas. ¿Acaso la preferencia por la disociación por encima de la sensibilidad del desarrollo evade a esta sensibilidad ética? Si no podemos escuchar las voces de los niños dentro de nosotros mismos y entre si, a la que nos sintoniza con el psicoanálisis del desarrollo, ¿cómo podremos escuchar los llantos de los hambrientos y destituidos? ¿Qué es lo que involucra la lucha por el desarrollo y la ética personal? Fácilmente puedo ver y escuchar como las partes de mí misma, llamadas "estados del self", debaten los pros y los contras de un problema político y de mi posible participación. Pero cuando digo: Heme aquí, no puedo hacer otra cosa. ¿Cómo es que la teoría de los estados del self se coloca para los gentiles justos, honrados en Yad Vashem, que arriesgaron todo para proteger a sus vecinos judíos? ¿Cómo describir a mi vecino activista, que va a prisión por protestarle al gobierno de Estados Unidos el trato a los inmigrantes indocumentados, o al psicólogo que confronta a los psicólogos involucrados en las torturas?

Nadie va a discutir el que uno necesita un total sentido unitario del self, mucho menos una etapa lineal de la teoría del desarrollo, para hacer estas cosas; al contrario, nos definimos como moral y cívicamente valientes en referencia a la capacidad para enfrentar nuestros miedos por el bien de algo que de verdad nos importa. Ahora quizá podríamos acercarnos al corazón del problema. El "Self" elude la definición, así como "identidad", muchos filósofos y psicólogos estarían de acuerdo, a través de la vida diaria nosotros los occidentales usamos estos conceptos constantemente e informalmente. Probablemente los necesitamos para nuestro discurso ${ }^{14}$ moral implícito. Cuando un paciente me dice que él o ella no tienen un self, o un no sentido del self, a veces pregunto, "¿Qué es lo que realmente importa para ti?" o "¿Qué es lo que más cuidas?" Después de cierto tiempo, si sale la respuesta, yo diría, bueno, esto es lo que es ser tú mismo, ser alguien que tiene un fuerte sentido de lo que importa para ti. Podemos construir con eso y ver que más hay ahí y que otras cosas te preocupan.

$\mathrm{Si}$, en todo caso, la respuesta viene a que en ciertos momentos él parece preocuparse por aquellos en su vida, y en otros momentos se comporta como si no existieran o importaran y no tuviera idea por qué, entonces ¿concluiría que tiene múltiples selfs, o, en cambio, que él (el locutor) está preocupado sobre algo seriamente mal en su vida por lo cual busca ayuda? Uno de mis primeros pacientes vino a mi porque ella encontró que alguien estaba tomando notas en sus clases con su letra, pero ella no tenía recuerdos de haber atendido a esas clases. Yo no concluí que ella tuviera múltiples selfs, pero entendí que ella (la paciente) era alguien que buscaba vivir como una persona. Ella no quería ser alguien con múltiples selfs, sino una 
persona integrada. Ella quería estar segura que podía ser responsable de todo lo que se ha hecho en su nombre.

¿Quién es el paciente en la teoría de los múltiples estados del self? ¿Habrá varios y varios analistas? Si un analista sostiene los supuestos de esta teoría, ¿qué ocurre con el paciente que llega buscando una mayor integración personal y ética? ¿podrá ella ser persuadida de abandonar su búsqueda? Para volver a nuestra pregunta anterior, ¿puede surgir un gran coraje personal de este tipo de pensamiento y tratamiento? ¿Estoy satisfecha, como paciente $o$ analista, de permanecer en los espacios entre mi estado del self en el que vivo con el derecho al privilegio blanco y que escucha las voces de Maya Angelou y Martin Luther King? ¿No debería luchar más allá de permanecer en los espacios?

Consideremos, por ejemplo, a Hans y Sophie Scholl. Estos estudiantes, miembros de la resistencia de White Rose, muertos en München en 1943, personificaban la empatía y serenidad que Heinz Kohut atribuyó a un sistema de valores completamente integrado. En una película reciente, Sophie Scholl: los días finales, basada en documentos recién descubiertos, me convenció de la justicia que Kohut hace sobre estos estudiantes. Este es el último sueño de Sophie: Después de haber sido despertada de su sueño para enfrentar el dia de su ejecución, ella dijo el siguiente sueño a su compañero de celda. En el sueño, ella decía, "era un día soleado, y yo llevaba un niño, vestido con un largo vestido blanco, para ser bautizado. El camino hacia la iglesia llevaba hacia una empinada montaña; pero yo llevaba al niño con firmeza y seguro. De pronto, se abrió una grieta frente a mí. Apenas tenía tiempo suficiente para depositar al niño lejos de la grieta, lo cual logré hacer con seguridad-después caí a las profundidades." (Kohut, 1985). "Después de que Sophie contara su sueño ella inmediatamente explicó su significado a su compañero. El niño, decía ella, es nuestra idea principal. Esta vivirá y hará su camino hacia la plenitud a pesar de los obstáculos" Kohut [p. 21]. Kohut (1985) dice más adelante: "Ella fue a su ejecución sin un rastro de miedo" (p.21). (La descripción de Winnicott de las condiciones suficientemente buenas y necesarias para el desarrollo de la capacidad de preocupación (Winnicott, 1965) apoya la perspectiva de Kohut aquí, creo yo).

Me parece que el énfasis de Kohut en el coraje civil como manifestación de la personalidad robusta deja en claro las implicaciones éticas de mi preocupación aquí. Sin hacer ninguna reivindicación metafísica para el self o de la identidad, parece importante expresar preocupación por el potencial evasivo en una teoría clínica que sugiere renunciar a la integración personal y a la integridad. O bien, siempre puedo decir, mi otro estado del self lo hizo, o no me dejo hacerlo. El propio Bromberg proporciona un maravilloso ejemplo personal de este proceso en su historia (P. M. Bromberg, 1996) sobre el hombre sin hogar que está fuera 
de su ventana y las dos tazas de café. Su diálogo interno, probablemente familiar para todos los que hemos vivido en ambientes urbanos, incluye una reflexión sobre la relevancia clínica de esta situación y como muchas de estas reflexiones, se queda corta. "iSalvados por la campana!" Nos quedamos con el self descentralizado, permitiendo la evasión de la pregunta ética. El coraje espera otro día. Por supuesto, uno podría objetar, los clínicos necesitan todo su coraje o valor sólo para enfrentar otro dia de sesiones. Es verdad, pero ¿nuestras teorías apoyan una creencia sobre el coraje último? ¿Nos preparan para actuar, como lo pensaron los pragmatistas, o solo para pararnos entre los espacios alternativos y de los estados del self?

La más reciente afirmación publicada por Bromberg (P. Bromberg, 2013), en honor a Stephen Mitchell, expresa en realidad un retorno integrado a sus puntos de vista anteriores sobre el psicoanálisis como un proceso de desarrollo, hacia la individualidad cohesiva. Utilizando a Winnicott, él escribe sobre la transformación de la fantasía en imaginación:

Para una persona que esta "imaginando", la situación es diferente; la persona está experimentando el self como existe ahora, proyectado hacia el futuro. Puesto que el self que se imagina es el mismo self que está creando la imaginación, la persona como es ahora tiene la capacidad de actuar en un futuro que es real pera él porque el futuro que se imagina en el aquí y ahora es real ( $\mathrm{pp}$ 12-13).

Aquí tenemos al psicoanálisis como un proceso de desarrollo, creando un sentido continuo del self que actúa, no contenido en permanecer en los espacios. Debemos esperar encarecidamente que los muchos usuarios y citadores de la teoría de los múltiples estados del self escuchen ahora a Philip Bromberg. Como él y como Stephen Mitchell, podemos encontrar el coraje para tomar nuestras teorías ligeramente.

\section{REFERENCIAS}

Aron, L. (2006). Analytic Impasse and the Third: Clinical implications of intersubjectivity Theory. International Journal of Psychoanalysis, 87, 349-368.

Aron, L., \& Bushra, A. (1998). Mutual Regression: Altered States in the Psychoanalytic Situation. J. Amer. Psychoanal. Assn., 46(2), 389-412.

Balint, M. (1992). The basic fault : therapeutic aspects of regression. Evanston, Ill.: Northwestern University Press.

Baraitser, L. (2008). Mum's the Word: Intersubjectivity, Alterity, and the Maternal Subject. Studies in Gender and Sexuality, 9, 86-110.

Bass, A. (2009). The As-If Patient and the As-If Analyst. Psychanalytic Quarterly, 76, 365-386.

Bass, G. (2002). Something Is Happening Here. Psychoanal. Dial., 12(5), 809-826.

Benjamin, J. (2004). Beyond Doer and Done to: An Intersubjective View of Thirdness. Psychoanalytic Quarterly, 73, 5-46. 
Birksted-Breen, D. (2003). Time and the après-coup. Int. J. Psycho-Anal. International Journal of Psychoanalysis, 84, 1501-1515.

Bromberg, P. (2009). Truth, Human Relatedness, and the Analytic Process: An Interpersonal/Relational Perspective. Int. J. Psycho-Anal. International Journal of Psychoanalysis, 90, 3347-3361.

Bromberg, P. (2011). The Gill/Bromberg Correspondence. Psychoanalytic Dialogues, 21, 243-252.

Bromberg, P. (2013). Hidden in Plain Sight: Thoughts on Imagination and the Lived Unconscious. Psychoanalytic Dialogues, 23, 1-14.

Bromberg, P. M. (1979). Interpersonal Psychoanalysis and Regression. Contemp. Psychoanal., 15, 647655.

Bromberg, P. M. (1991). On Knowing One's Patient Inside Out: The Aesthetics of Unconscious Communication. Psychoanal. Dial., 1(4), 399-422.

Bromberg, P. M. (1994). "Speak! That I May See You": Some Reflections on Dissociation, Reality, and Psychoanalytic Listening. Psychoanal. Dial., 4(4), 517-547.

Bromberg, P. M. (1996). Standing in the Spaces: The Multiplicity Of Self And The Psychoanalytic Relationship. Contemp. Psychoanal., 32, 509-535.

Bromberg, P. M. (1998). Standing in the spaces : essays on clinical process, trauma, and dissociation. Hillsdale, NJ: Analytic Press.

Chefetz, R. (2003). Healing haunted hearts: toward a model for integrating subjectivity, commentary on papers by Philip Bromberg and Gerald Stechler. Psychoanalytic Dialogues, 13(727-742).

Chefetz, R., \& Bromberg, P. (2004). Talking with "Me" and "Not-Me": A Dialogue. Contemporary Psychoanalysis, 40(409-464).

Cooper-White, P. (2007). Many voices : pastoral psychotherapy in relational and theological perspective. Minneapolis, MN: Fortress Press.

Cushman, P. (2007). A Burning World, an Absent God: Midrash, Hermeneutics, and Relational Psychoanalysis. Contemp. Psychoanal., 43, 47-88.

Dahl, G. (2010). Nachträglichkeit, Wiederholungszwang, Symbolisierung: Zur psychoanalytischen Deutung von primärprozesshaften Szenen. Psyche Zeitung für Psychoanalyse, 64 385-407.

Davies, J. (2009). Love Never Ends Well: Termination as the Fate of an Illusion: Commentary on Papers by Jill Salberg and Sue Grand. Psychoanalytic Dialogues, 19, 734-743.

Davies, J. M. (1996). Linking the "Pre-Analytic" with the Postclassical: INTEGRATION, DISSOCIATION, AND THE MULTIPLICITY OF UNCONSCIOUS PROCESS. Contemp. Psychoanal., 32, 553-576.

Ferenczi, S. (1931). Child-Analysis in the Analysis of Adults. Int. J. Psycho-Anal., 12, 468-482.

Ferenczi, S. (1949). Confusion of the Tongues Between the Adults and the Child-(The Language of Tenderness and of Passion). Int. J. Psycho-Anal., 30, 225-230.

Ferenczi, S., \& Dupont, J. (1988). The clinical diary of Sándor Ferenczi. Cambridge, Mass.: Harvard University Press.

Freud, S. (1917). Introductory Lectures on Psycho-Analysis: 27th and 28th Lectures. Standard Edition (Vol. 16-17).

Freud, S., \& Strachey, J. (2005). Civilization and its discontents. New York: Norton.

Friedman, L. (2008). Loewald. J. Amer. Psychoanal. Assn., 56, 1105-1115.

Gerson, S. (2009). When the Third is Dead: Memory, Mourning, and Witnessing in the Aftermath of the Holocaust. International Journal of Psychoanalysis, 90, 1341-1357.

Grand, S. (2000). The reproduction of evil : a clinical and cultural perpsective. Hillsdale, NJ: Analytic Press. Grand, S. (2010). The hero in the mirror : from fear to fortitude. New York: Routledge.

Greenberg, J. R., \& Mitchell, S. A. (1983). Object relations in psychoanalytic theory. Cambridge, Mass.: Harvard University Press. 
Harris, A. (1996). The Conceptual Power of Multiplicity. Contemp. Psychoanal., 32, 537-552.

Harris, A. (2011). The relational tradition: landscape and canon. Journal of the American Psychanalytic Association, 59, 701-735.

Harris, A., \& Bass, A. (2011). Nachtraglichkeit. Psychoanalytic Dialogues, 21, 239-242.

Harris, A., \& Suchet, M. (2002). Relationality: From Attachment to Intersubjectivity. Stephen A. Mitchell. Hillsdale, NJ: Analytic Press, 2000. xvii \& 173 pp. \$39.95. American Imago, 59, 102-111.

Hirsch, I. (1997). The Widening of the Concept of Dissociation. J. Amer. Acad. Psa., 25(4), 603-615.

Hoffman, I. Z. (2000). At Death's Door. Psychoanal. Dial., 10(6), 823-846.

Knoblauch, S. H. (1999). The Third, Minding and Affecting. Psychoanal. Dial., 9(1), 41-51.

Kohut, H. (1985). On Courage. In C. Strozier \& E. Kohut (Eds.), Self Psychology and the Humanities (pp. 5-50). New York: Norton.

Lachmann, F. M. (1996). How Many Selves Make a Person? Contemp. Psychoanal., 32, 595-614.

Layton, L. (2009). Who's Responsible? Our Mutual Implication in Each Other's Suffering. Psychoanalytic Dialogues, 19, 105-120.

Lear, J. (1998). Love and its place in nature : a philosophical interpretation of Freudian psychoanalysis. New Haven, CT: Yale University Press.

Leary, K. (2000). Racial Enactments in Dynamic Treatment. Psychoanal. Dial., 10(4), 639-653.

Levi, P. (1988). The drowned and the saved. New York: Summit Books.

Loewald, H. (2007). Internalization, Separation, Mourning, and the Superego. Psychoanlytic Quarterly, $76,1113-1133$.

Loewald, H. W. (1960). On the Therapeutic Action of Psycho-Analysis. Int. J. Psycho-Anal., 41, 16-33.

Loewald, H. W. (1985). Oedipus Complex and Development of Self. Psychoanal O., 54, 435-443.

Loewald, H. W., Loewald, H. W., Loewald, H. W., \& Loewald, H. W. (2000). The essential Loewald: collected papers and monographs. Hagerstown, Md.: University Pub. Group.

Mitchell, S. (2004). My Psychoanalytic Journey. Psychoanalytic Inquiry, 24, 531-541.

Mitchell, S. A. (1979). Twilight of the Idols-Change and Preservation in the Writings of Heinz Kohut. Contemp. Psychoanal., 15, 170-189.

Mitchell, S. A. (1983). Reflections. Contemp. Psychoanal., 19, 133-139.

Mitchell, S. A. (1984). Object Relations Theories and the Developmental Tilt. Contemp. Psychoanal., 20, 473-499.

Mitchell, S. A. (1987). Discussion. Contemp. Psychoanal., 23, 400-409.

Mitchell, S. A. (1988). Relational concepts in psychoanalysis : an integration. Cambridge, Mass.: Harvard University Press.

Mitchell, S. A. (1993). Hope and dread in psychoanalysis. New York: BasicBooks.

Mitchell, S. A. (1997). Influence and autonomy in psychoanalysis. Hillsdale, NJ: Analytic Press.

Mitchell, S. A. (1998). From Ghosts to Ancestors: The Psychoanalytic Vision of Hans Loewald. Psychoanal. Dial., 8(6), 825-855.

Mitchell, S. A. (2000). Relationality: from attachment to intersubjectivity. Hillsdale, NJ: Analytic Press.

Muller, J. P. (1999). The Third as Holding the Dyad. Psychoanal. Dial., 9(4), 471-480.

Ogden, T. H. (1986). The matrix of the mind : object relations and the psychoanalytic dialogue. Northvale, N.J.: J. Aronson.

Ogden, T. H. (1994). The Analytic Third: Working with Intersubjective Clinical Facts. Int. J. Psycho-Anal., $75,3-19$.

Orange, D. (2013). Those Old Wineskins: Greenberg and Mitchell on Heinz Kohut's "Mixed Model". . Contemp. Psychoanal., 49, 103-112.

Orange, D. (2016a). Climate Crisis, Psychoanalysis, and Radical Ethics. London: Routledge. 
Orange, D. (2016b). Nourishing the Inner Life of Clinicians and Humanitarians: the Ethical Turn in Psychoanalysis. London and New York: Routledge.

Orange, D. M. (2011). The suffering stranger : hermeneutics for everyday clinical practice. New York: Routledge/Taylor \& Francis Group.

Peirce, C. (1998). The Essential Peirce (Vol. 2): Indiana University Press.

Putnam, F. W. (1988). The switch process in multiple personality disorder and other state-change disorders. Dissociation. Dissociation, 1, 24-32.

Racker, E. (1968). Transference and counter-transference. New York, International Universities Press.

Riker, J. H. (2013). The Philosophical Importance of Kohut's Notion of the Self. International Journal of Psychoanalytic Self Psychology, 8, 495-504.

Sander, L. W. (1995). Identity and the Experience of Specificity in a Process of Recognition: Commentary on Seligman and Shanok. Psychoanal. Dial., 5(4), 579-593.

Shaw, D. (2003). On the Therapeutic Action of Analytic Love. Contemporary Psychoanalysis, 39, 251-278.

Shaw, D. (2014). Traumatic narcissism : relational systems of subjugation. New York, NY: Routledge.

Shengold, L. (1989). Soul murder : the effects of childhood abuse and deprivation. New Haven: Yale University Press.

Slochower, J. A. (2013). Holding and psychoanalysis : a relational perspective (2nd edition. ed.). Hove, East Sussex: Routledge.

Stern, D. N. (1985). The interpersonal world of the infant : a view from psychoanalysis and developmental psychology. New York: Basic Books.

Stolorow, R. D. (2007). Trauma and human existence : autobiographical, psychoanalytic, and philosophical reflections. New York: Analytic Press.

Sullivan, H. S., \& Mullahy, P. (1948). Conceptions of modern psychiatry. Washington,

Taylor, C. (1989). Sources of the self : the making of the modern identity. Cambridge, Mass.: Harvard University Press.

Thelen, E., \& Smith, L. (1996). A Dynamic Systems Approach to the Development of Cognition and Action Cognitive Psychology Ser Retrieved from http://www.columbia.edu/cgi-bin/cul/resolve?clio76g0076

Winnicott, D. W. (1965). The Maturational Processes and the Facilitating Environment (Vol. 64).

Wittgenstein, L. (1953/2001). Philosophical Investigations (G. E. M. Anscombe, Trans. Third, with revised English translation ed.). Oxford: Blackwell.

Yerushalmi, H. (2001). Self-States and Personal Growth in Analysis. Contemp. Psychoanal., 37(3), 471488.

Original recibido con fecha: 21-6-2016 Revisado: 23-6-2016 Aceptado: 30/06/2017

NOTAS:

${ }^{1}$...no importa que tan lejos la ciencia vaya, aquellas inferencias que predominan en la mente del investigador son bastante inciertas. Están a prueba. Éstas deben tener un juicio justo y no ser condenadas hasta ser probadas falsas más allá de toda duda; y en el momento en que la prueba es alcanzada, el investigador debe estar listo para abandonarlas sin el menor cariño hacia ellas. Así, el investigador científico tiene que estar siempre dispuesto, en un momento, a abandonar de inmediato todas las teorías al estudio de las que ha estado dedicando quizá muchos años. (Peirce, 1998), p. 25.

${ }^{2}$ Aquí tomo prestado de mi discusión sobre el punto de vista de Greenberg y Mitchell sobre Kohut (D. Orange, 2013). 


\footnotetext{
3 Sería más que injusto el ignorar el uso que Thomas Ogden (Thomas H. Ogden, 1986) hace de la matriz. Su uso de ella sugiere su referencia etimológica hacia la madre y particularmente se refiere a Winnicott. No sé si Mitchell y Ogden llegaron a sus usos de esta palabra de manera independiente, pero sospecho que sus usos podrían haber influido el uno al otro.

4 Este apartado expande párrafos sobre Loewald en (D. Orange, 2016b).

5 En Ferenczi (S. Ferenczi, 1931; Sándor Ferenczi \& Dupont, 1988) se observa una reverencia similar por el proceso regresivo necesario, tan influyente en el psicoanálisis contemporáneo (cf. (Arn \& Bushra, 1998), así como en las creativas confrontaciones con el mal en la obra de Sue Grand (Grand, 2000, 2010) donde perpetradores y sufrientes se reenganchan, extrañamente.
}

${ }^{6} \mathrm{~N}$. de T. se ha traducido la palabra puzzle por rompecabezas, que se define como un juego que consiste en componer determinada figura combinando cierto número de piezas de madera o cartón, en cada uno de los cuales hay una parte de la figura.

7 El por qué Loewald ha sido tan ignorado es otra pregunta, formulada por Lawrence Friedman (Friedman, 2008). Harris y Suchet (Harris \& Suchet, 2002) observan claramente esta influencia en su revisión de (S. A. Mitchell, 2000) así como lo hace (Harris, 2011).

${ }^{8} \mathrm{~N}$. de T. la traducción es mía, el título original es Sources of the Self (Taylor, 1989).

9 Reemplazando los conceptos unívocos que se aplican de la misma manera a todo lo que abarcan y reemplazando las categorías a las cuales las personas o cosas simplemente pertenecen o no, nos recuerda que, en las familias, algunos miembros tienen ojos similares, otros tienen narices, mentones o cabellos similares. Estas características, pensó, nos permiten reconocer a las personas como parecidas incluso cuando no hay dos de ellas que tengan las mismas características en común. Pensaba que los conceptos, tanto de colores como de teorías, se superponían de manera similar. "Vemos una complicada red de similitudes superpuestas y entrecruzadas: a veces similitudes generales, a veces similitudes de detalle" (Wittgenstein, 1953/2001), párrafo 66).

${ }^{10}$ Es interesante resaltar que la expresión "estado del self" se volvió prominente en el trabajo de Heinz Kohut sobre sueños del estado del self y después fue retomado por Stern y Sander en sus estudios de desarrollo. Ellos consideraban que el trabajo del psicoanálisis debería preocuparse por la transformación de los estados del self. ${ }^{11}$ Pido perdón si debí de haber citado a otros; estos son ejemplos.

${ }^{12}$ Uno de mis pacientes más seriamente "disociativos" se opuso repetidamente a mí que la "disociación" es un nombre inapropiado; Uno más bien se asocia, bajo las condiciones relacionales correctas, al pasado traumático. ${ }^{13} \mathrm{~N}$. del T. La traducción del término alemán Nachträglichkeit en inglés la definen como afterwardness y en español la he traducido por "acción diferida".

${ }^{14} \mathrm{El}$ postmodernismo ha estado en cuestión, por no decir en desaparición, en los últimos años a causa de los problemas éticos de sus más grandes intelectuales: Heidegger, Paul de Man y tal vez incluso Derrida. Por lo tanto, no es de extrañar que un psicoanálisis dependiente de las ideas postmodernas también pudiera fundar sobre bancos éticos. Puede abarcar la diferencia, pero no saber cómo enfrentarse a los totalitarios. 